\title{
$9-2018$
}

\section{Invasive community acquired methicillin-resistant staphylococcal aureus (CA-MRSA) infections in children}

\author{
Muhammad Khalid \\ Aga Khan University \\ Samina Junejo \\ Aga Khan University \\ Fatima Mir \\ Aga Khan University, fatima.mir@aku.edu
}

Follow this and additional works at: https://ecommons.aku.edu/

pakistan_fhs_mc_women_childhealth_paediatr

Part of the Pediatrics Commons

\section{Recommended Citation}

Khalid, M., Junejo, S., Mir, F. (2018). Invasive community acquired methicillin-resistant staphylococcal aureus (CA-MRSA) infections in children. Journal of the College of Physicians and Surgeons--Pakistan, 28(9), S174-S177.

Available at: https://ecommons.aku.edu/pakistan_fhs_mc_women_childhealth_paediatr/786 


\title{
Invasive Community Acquired Methicillin-Resistant Staphylococcal Aureus (CA-MRSA) Infections in Children
}

\author{
Muhammad Khalid, Samina Junejo and Fatima Mir
}

\begin{abstract}
Staphylococci are gram-positive bacteria divided into coagulase positive and coagulase negative classes, Staphylococcus aureus is the most important bacterium of this class. Epidemiology of methicillin-resistant Staphylococcus aureus (MRSA) has changed a lot. It is no more the problem of only hospitalised patients. Children coming from community has also been increasingly affected by MRSA-called community acquired methicillin-resistant (CA-MRSA) infection. The higher severity of CA-MRSA is due to its ability to produce the toxin Panton-Valentine Leukocidin (PVL) associated with staphylococcal cassette chromosome mec (SCCmec) type IV gene. Here, we are presenting five cases of CA-MRSA infection in children having age range 0.5 months to 11 years. All of them had invasive MRSA infection finally diagnosed as causing empyema thoracis, infective endocarditis, psoas abscess and necrotising fasciitis. Early surgical intervention, quick microbiological recognition of the pathogen, and appropriate antimicrobial therapy helped save their lives.
\end{abstract}

Key Words: Methicillin-resistant Staphylococcus aureus. Pleural empyema. Panton-valentine leukocidin. Necrotising fasciitis.

\section{INTRODUCTION}

Staphylococci are normal human flora found in the nose, throat, skin and intestine. These bacteria can invade the human system causing toxin mediated food poisoning, skin and soft tissue infections, pneumonia and bacteremia. Methicillin-resistant Staphylococcal aureus (MRSA) infection is a well-recognised cause of hospital acquired infections in the world. Community acquired MRSA (CA-MRSA) are non-multiresistant, and children are more likely to have community than the hospital acquired MRSA. Skin and soft tissue infections due to Staphylococcus aureus are common in children. But invasive CA-MRSA (deep abscesses, meningitis, osteomyelitis and infective endocarditis) infections in children are rare. ${ }^{1}$

This report described five cases of severe CA-MRSA infection in children admitted at The Aga Khan University Hospital, Karachi between 2015 - 2016.

\section{CASE REPORT}

Case 1: A two-month infant presented with high grade fever and respiratory difficulty for three days. His work of breathing increased and had spiking fever despite antipyretics and antibiotics (amoxicillin) prescribed by local pediatrician two days ago. He was born full term through normal delivery with no postnatal problem.

Department of Pediatric Infectious Diseases, The Aga Khan University Hospital, Karachi.

Correspondence: Dr. Fatima Mir, Assistant Professor,

Department of Pediatric Infectious Diseases, The Aga Khan

University Hospital, Stadium Road, Karachi.

E-mail:fatima.mir@aku.edu

Received: August 08, 2017; Accepted: February 21, 2018.
On examination, he was febrile, heart rate of 135 /minute, respiratory rate of $40 /$ minute, and oxygen saturation $\left(\mathrm{SpO}_{2}\right)$ in room air was $98 \%$. The left side of the chest was bulging with decreased air entry on auscultation. His chest X-ray showed complete white - out in the left hemithorax with deviation of the trachea towards right side. Due to mediastinal shift, chest tube was placed by cardiothoracic surgery and child was shifted to pediatric intensive care unit (PICU); and broad spectrum antibiotics (I/V meropenem and vancomycin) were started. Thick pus obtained on chest intubation was sent for culture sensitivity.

Due to persisting fever and tachypnea, CT-chest was obtained the next day. It showed left-sided empyema. Patient underwent VATS and decortication with two chest tubes placed on left side. After 48 hours, the pus culture grew MRSA. Patient was shifted to intravenous vancomycin only, and this condition gradually improved, so he was shifted to general ward. He was discharged, after three weeks of hospital admission, on oral linezolid to complete six weeks therapy.

Case 2: An 11-year boy presented with pain in abdomen and fever for four days. It was not associated with loose motion, vomiting or any urinary complaints. Abdominal examination revealed significant tenderness in right lower abdomen with restricted movements of right leg. Remaining systemic examination was unremarkable. An initial impression of acute appendicitis was made. Initial laboratory work-up of the patient included CBC, blood culture, urine DR and culture and ultrasound of abdomen.

Ultrasound (USG) of abdomen revealed right iliopsoas abscess with pus volume of approximately $50 \mathrm{cc}$. There was no evidence of abscess in the left iliopsoas region or acute appendicitis. Work up for tuberculosis-chest X-ray, Mantoux test and pus for AFB staining and 
culture, remained negative. The boy underwent USG guided drainage of pus $(45 \mathrm{ml})$ and pigtail catheter placement. Pus was sent for culture and sensitivity. Pus culture showed MRSA. Patient received I/V vancomycin for two weeks and was discharged on oral clindamycin and linezolid for three more weeks. Catheter was removed on follow-up when there was no residual collection on USG abdomen.

Case 3: A five-month infant was referred due to rapidly progressive and deteriorating condition from a secondary care hospital. She was admitted as she had one week history of fever and cough for which she was given antibiotics from local doctor with no improvement. On examination, she was looking sick, irritable with inconsolable cry, respiratory rate $50 /$ minute, and febrile to touch. $\mathrm{Her} \mathrm{SpO}_{2}$ was $91 \%$ at $4 \mathrm{~L} / \mathrm{min}$ oxygen. The area around her left eye was also swollen. Her chest X-ray showed right-sided hydropneumothorax and an illdefined opacity seen in the right lung, suggestive of infection (Figure 1). I/V line was maintained, sepsis work-up (CBC, CRP, blood cultures, CSF DR and C/S) was sent and child started on broad spectrum antibiotics, meropenem and vancomycin.

Her CT (chest) findings were consistent with empyema thoracis (Figure 1). CT (brain and orbit) showed left frontal, pre-septal region and forehead collections along with subdural empyema. CSF examination was consistent with acute bacterial meningitis. The child underwent VATS with chest tube placement and drainage of the pre-septal and subdural collections. All specimen obtained were sent for culture and sensitivity. The cultures showed heavy growth of Staphylococcus aureus that was same in all specimens and had same antibiotic sensitivities.

The child had issues of maintaining vancomycin levels; for which, Hickman line was placed and continuous vancomycin infusion was started. The child gradually improved over a period of four weeks when chest tubes were successfully removed. The child completed six weeks of intravenous vancomycin with monitoring of renal functions which remained within normal limits. Due to extensive disease nature, immunodeficiency work up was done later, which remained negative.

Case 4: An 11-year girl presented with fever for one week, body rash for 3 days, and altered state of consciousness for one day. She was in her good state of health when she developed sudden high grade, continuous fever, followed by generalised purpuric rash on body. These were associated with breathlessness and mild cough. She was getting intravenous ceftriaxone and amikacin for three days; but her consciousness level worsened, so she was referred to us. She was previously not diagnosed with congenital heart disease or rheumatic heart disease.

On examination, she was obtunded (GCS 8/15), tachycardiac (150/minutes), hypotensive $(90 / 50 \mathrm{~mm}$ of $\mathrm{Hg})$,

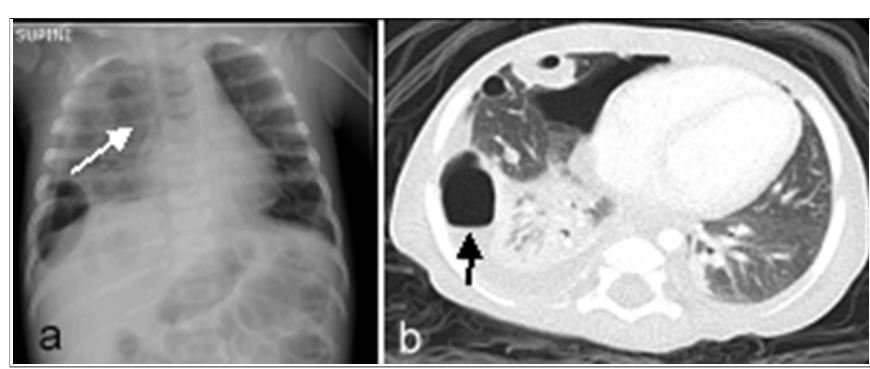

Figure 1: (a) Chest X-ray; (b) CT scan chest showing the infiltrates and empyema.

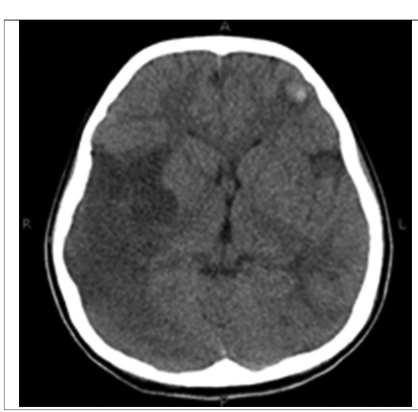

Figure 2: CT-scan brain showing multifocal infarcts.

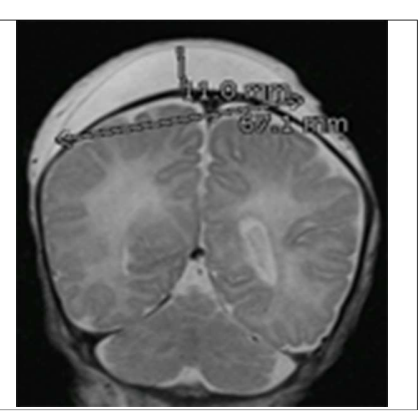

Figure 3: MRI-brain showing the scalp collection. respiratory rate of $40 /$ minute, and capillary refill time $>3 \mathrm{sec}$. Abdominal examination revealed hepatosplenomegaly, gangrenous fingers of hands and feet. Cardiovascular examination showed normal first heart sound, single second heart sound with grade 3/6 pansystolic murmur at apical region. Endotracheal intubation done and she was managed as septic shock/ acute infective endocarditis with embolic phenomena, with $\mathrm{I} / \mathrm{V}$ lines maintained, sepsis work-up sent, fluid resuscitation done and broad spectrum antibiotics (ceftriaxone, vancomycin) started; and patient shifted to PICU. Her initial CT scan brain showed multifocal infarcts involving the right fronto-parietal and left frontal lobe (Figure 2). Her echocardiography showed thickened mitral valve with prolapse, moderate eccentric mitral regurgitation and large mobile vegetation attached on anterior mitral leaflet measuring $27 \times 12 \mathrm{~mm}$ in size, mildly dilated left ventricle.

Her three blood cultures collected from different sites grew Staphylococcus aureus. Patient was shifted to vancomycin and gentamicin, according to infective endocarditis protocol. Once stabilised, surgical removal of vegetation and mitral valve replacement was done. Culture of the vegetation also grew MRSA. She was extubated 48 hours later, shifted to step down and monitored for appropriate vancomycin trough levels. She was successfully discharged three weeks later on $\mathrm{I} / \mathrm{V}$ vancomycin to complete six week therapy.

Case 5: A 15-day male neonate was brought for fever and swelling over the head. The swelling had started 5 days earlier, progressed gradually and now developed necrosis of the skin with fever. The baby was born full term, appropriate for gestational age by cesarean 
Table I: Final diagnosis and laboratory work-up of cases with CA-MRSA infection.

\begin{tabular}{|c|c|c|c|c|}
\hline Age/gender & Final diagnosis & Hematological work-up & $\begin{array}{l}\text { Antimicrobial sensitivities } \\
\text { (MRSA) }\end{array}$ & Radiological findings \\
\hline 2-month/M & Left lung empyema & $\begin{array}{l}\mathrm{Hb}-10.5 \mathrm{~g} / \mathrm{dl} \\
\mathrm{TLC}-21,000 / \mathrm{mm} 3 \\
\text { Platelets-1,383,000/mm3 } \\
\text { CRP - } 8.95 \\
\text { Procalcitonin - } 0.55\end{array}$ & $\begin{array}{l}\text { Sensitive to clindamycin, } \\
\text { tetracycline, linezolid, } \\
\text { vancomycin }\end{array}$ & $\begin{array}{l}\text { CT - chest - complete collapse of left lung and } \\
\text { collection approximately measuring } 63 \times 50.2 \times 70.6 \mathrm{~mm} \\
\text { in AP, transverse and craniocaudal dimensions. } \\
\text { Atelectasis was identified in the right middle and lower } \\
\text { lobes. }\end{array}$ \\
\hline 11-year/M & Rt. Iliopsoas abscess & $\begin{array}{l}\mathrm{Hb}-9.8 \mathrm{~g} / \mathrm{dl} \\
\mathrm{TLC}-13,000 / \mathrm{mm} 3 \\
\text { Platelets - 292,000/mm3 } \\
\text { ESR / CRP - 62/29.39 }\end{array}$ & $\begin{array}{l}\text { Sensitive to clindamycin, } \\
\text { co-trimoxazole, vancomycin }\end{array}$ & $\begin{array}{l}\text { Ultrasound (USG) abdomen showed irregular } \\
\text { shaped, lobulated, echogenic focus identified within } \\
\text { the right iliopsoas muscle representing iliopsoas } \\
\text { abscess. It measured } 85 \times 37 \times 32 \text { mm in CC, AP \& } \\
\text { TS dimensions respectively with volume of } \\
\text { approximately } 50 \mathrm{cc} \text {. }\end{array}$ \\
\hline 5-month/F & $\begin{array}{l}\text { Rt. lung empyema, left eye } \\
\text { pre-septal cellulitis and } \\
\text { subdural empyema with } \\
\text { meningitis }\end{array}$ & $\begin{array}{l}\mathrm{Hb}-8.3 \mathrm{~g} / \mathrm{dl} \\
\mathrm{TLC}-46,000 / \mathrm{mm} 3 \\
\text { Platelets }-934,000 / \mathrm{mm} 3 \\
\text { CRP - } 32.28 \\
\text { Procalcitonin - } 7.39\end{array}$ & $\begin{array}{l}\text { Sensitive to gentamicin, } \\
\text { clindamycin, erythromycin, } \\
\text { vancomycin, and } \\
\text { levofloxacin }\end{array}$ & $\begin{array}{l}\text { CT - chest - hydropneumothorax on right side, multiple } \\
\text { thick walled cavities in the lung fields bilaterally } \\
\text { predominantly in the lower lobes, nodular density } \\
\text { representing consolidation is noted in left lower lobe } \\
\text { (Figure 1). } \\
\text { CT - brain and orbit - low attenuating swelling } \\
\text { identified in left frontal and pre-septal region likely } \\
\text { representing small collections measuring } 21 \times 15 \times 9.0 \mathrm{~mm} \\
\text { and forehead collection measures } 22 \times 14 \times 5.0 \mathrm{~mm} \text {, } \\
\text { prominent subdural spaces seen with fluid of high } \\
\text { attenuation suggesting subdural empyema. }\end{array}$ \\
\hline $11-$ Year/F & $\begin{array}{l}\text { Infective endocarditis with } \\
\text { embolic phenomena }\end{array}$ & $\begin{array}{l}\mathrm{Hb}-8.5 \mathrm{~g} / \mathrm{dl} \\
\mathrm{TLC}-39,000 / \mathrm{mm} 3 \\
\text { Platelets }-340,000 / \mathrm{mm} 3 \\
\text { CRP - } 17.37 \\
\text { Procalcitonin - } 22\end{array}$ & $\begin{array}{l}\text { Sensitive to gentamicin, } \\
\text { clindamycin, erythromycin, } \\
\text { levofloxacin, vancomycin } \\
\text { and rifampicin }\end{array}$ & $\begin{array}{l}\text { CT - scan brain - multifocal infarcts involving the right } \\
\text { fronto-parietal and left frontal lobe (Figure } 2 \text { ). }\end{array}$ \\
\hline 15-day/M & Necrotizing fasciitis & $\begin{array}{l}\mathrm{Hb}-8.3 \mathrm{~g} / \mathrm{dl} \\
\mathrm{TLC}-27,500 / \mathrm{mm} 3 \\
\text { Platelets - } 136,000 / \mathrm{mm} 3 \\
\text { CRP - } 34.78\end{array}$ & $\begin{array}{l}\text { sensitive to gentamicin, } \\
\text { clindamycin, erythromycin, } \\
\text { vancomycin and linezolid }\end{array}$ & $\begin{array}{l}\text { MRI brain - large scalp collection occupying almost } \\
\text { whole of the vertex and extending into the preseptal } \\
\text { region appearing as hypointense on T1-weighted and } \\
\text { hyperintense on T2-weighted sequences. It measured } \\
\text { approximately } 7 \times 11 \times 1.1 \mathrm{~cm} \text { in transverse AP and } \\
\text { craniocaudal dimensions. No evidence of intracranial } \\
\text { extension is identified (Figure } 3 \text { ). }\end{array}$ \\
\hline
\end{tabular}

section with no postnatal complications and was discharged home on second day of life. There was no history of trauma; and baby was given I/M vit. $\mathrm{K}$ at birth. On examination, baby was febrile but vitally stable. There was large swelling extending from vertex to preseptal region, overlying skin was necrotic and there was serosanguineous discharge from the collection. Fluid sample was sent for culture sensitivity. MRI brain was done to study intracranial extent which showed large scalp collection occupying almost whole of the vertex and extending into the preseptal region. No evidence of intracranial extension was identified. Sepsis work-up was sent and broad spectrum antibiotics were started.

Patient underwent wound debridement and tissue samples were sent for culture sensitivity. Blood cultures remained negative but fluid and tissue c/s grew moderate growth of MRSA. Intravenous vancomycin was continued with daily dressing. After three weeks of antibiotic therapy and clean wound, baby had split segment grafting (SSG) of the wound by plastic surgery team. The graft was successfully taken-up and patient was discharged home.

\section{DISCUSSION}

MRSA is resistant to penicillin and cephalosporins. This resistance is mediated by penicillin binding protein $2 a$, which is encoded by mecA gene. This mecA gene is situated in SCCmec, which is type II in case of hospital acquired MRSA. ${ }^{2}$ First reported in 1990, CA-MRSA carries SCCmec type V or IV. It may be associated with a toxin Panton-Valentine Leukocidin (PVL). Clinical isolates carrying PVL cause more severe infections and necrotising pneumonia. The common risk factors for MRSA infection include surgery, invasive devices, dialysis, hospital admission in previous 12 months and colonisation with MRSA. ${ }^{3}$

None of these patient had any known risk factor for MRSA infection. Young age, poor hygiene, overcrowding leading to close contacts in community and horizontal transmission of resistant genes are some of the proposed factors for invasive CA-MRSA infection in children. Three of these cases were below one year of age and they did not have hospitalisation apart from routine vaccination visits. A cross-sectional study conducted by Reta et al. in Ethopian school children found $13.8 \%$ nasal carriage of MRSA. 4

A wide spectrum of clinical disease is described with CA-MRSA, most common of which is skin and soft tissue infections (SSTI-furuncle, carbuncle, and cellulitis). However severe invasive CA-MRSA infections are also described. These include lung abscess, endocarditis, 
brain abscess, myositis, osteomyelitis and iliopsoas abscess. ${ }^{2}$ All these patients presented with invasive disease involving lungs, skin and soft tissues (necrotising fasciitis and psoas abscess), meningitis and infective endocarditis.

CA-MRSA isolates are sensitive to clindamycin, trimethoprim-sulphamethoxazole, rifampicin, fluoroquinolones, tetracycline, vancomycin and linezolid. 5 All these patients were resistant to methicillin but susceptible to clindamycin. Clindamycin sensitivity in the microbiology laboratory is reported only after performing D-test. Erythromycin and clindamycin discs are placed adjacent to each other on culture plate, appearance of 'D-zone' indicates inducible clindamycin resistance (D-test). ${ }^{6}$

The optimal treatment is yet not established for these isolates. In 2014, Infectious Disease Society of America (IDSA) has recommended the use of vancomycin or linezolid. ${ }^{7}$ Apart from antimicrobial therapy, surgical drainage of the infected collections hastens the recovery. Radiological imaging provides useful adjunct to assess the extent and severity of disease. These patients received at least 2-3 weeks intravenous vancomycin before they were shifted to oral linezolid. In case of infective endocarditis, 6-week therapy with vancomycin was completed. Vancomycin trough levels were monitored in all patients and maintained at 10-15 $\mu \mathrm{g} / \mathrm{ml}$. All patients had successful recovery and are doing well on follow-up.

CA-MRSA infection in children is rapidly progressive resulting in bacteremia, meningitis, necrosis, and abscesses formation. Surgical drainage and debridement not only help in controlling the source but also provide specimen for microbiological diagnosis for appropriate antimicrobial therapy. Intravenous vancomycin (with monitoring of blood levels) should be first line of therapy in such cases, followed by oral antibiotics (clindamycin, levofloxacin, cotrimoxazole and linezolid), according to antibiotic sensitivity report to complete the treatment duration. Educating patients about hand hygiene and environmental cleaning can be the effective strategies in preventing CA-MRSA infections.

\section{REFERENCES}

1. Alrabiah K, Al Alola S, Al Banyan E, Al Shaalan M, Al Juhani S. Characteristics and risk factors of hospital acquired-Methicillinresistant Staphylococcus aureus (HA-MRSA) infection of pediatric patients in a tertiary care hospital in Riyadh, Saudi Arabia. Int J Adolesc Med Health 2016; 3:71-7.

2. Qiao $Y$, Ning $X$, Chen $Q$, Zhao $R$, Song $W$, Zheng $Y$, et al. Clinical and molecular characteristics of invasive communityacquired Staphylococcus aureus infections in Chinese children. BMC Infect Dis 2014; 14:1.

3. Fukuta Y, Cunningham CA, Harris PL, Wagener MM, Muder RR. Identifying the risk factors for hospital-acquired methicillinresistant Staphylococcus aureus (MRSA) infection among patients colonized with MRSA on admission. Infect Control Hosp Epidemiol 2012; 33:1219-25.

4. Reta A, Gedefaw L, Sewunet T, Beyene G. Nasal carriage, risk factors and antimicrobial susceptibility pattern of methicillin resistant Staphylococcus aureus among school children in Ethiopia. J Med Microb Diagn 2015; 4:2.

5. Abbas A, Nirwan P, Srivastava P. Prevalence and antibiogram of hospital acquired-methicillin resistant Staphylococcus aureus and community acquired-methicillin resistant Staphylococcus aureus at a tertiary care hospital National Institute of Medical Sciences. Communit Acquir Infect 2015; 2:13.

6. Ghosh S, Banerjee M. Methicillin resistance \& inducible clindamycin resistance in Staphylococcus aureus. Indian $J$ Med Res 2016; 143:362.

7. Stevens DL, Bisno AL, Chambers HF, Dellinger EP, Goldstein EJ, Gorbach SL, et al. Practice guidelines for the diagnosis and management of skin and soft tissue infections: 2014 update by the Infectious Diseases Society of America. Clin Infect Dis 2014; 59:e10-e52. 